\title{
AHP FOR HEALTH TECHNOLOGY ASSESSMENT. A CASE STUDY: PRIORITIZING CARE APPROACHES FOR PATIENTS SUFFERING FROM CHRONIC HEART FAILURE
}

\author{
Leandro Pecchia* \\ Department of Biomedical, Electronic and Telecommunication Engineering, \\ University Federico II of Naples \\ Naples, Italy \\ E-mail: leandro.pecchia@unina.it \\ Umberto Bracale \\ Department of vascular and thoracic surgery, University Federico II of Naples. Italy. \\ University Federico II of Naples \\ Napoli, Italy \\ E-mail: reumbe@tin.it \\ Paolo Melillo \\ Department of Biomedical, Electronic and Telecommunication Engineering, \\ University Federico II of Naples \\ Napoli, Italy \\ E-mail: paolo.melillo@unina.it \\ Mario Sansone \\ Department of Biomedical, Electronic and Telecommunication Engineering, \\ University Federico II of Naples \\ Napoli, Italy \\ E-mail: msansone@unina.it \\ Marcello Bracale \\ Department of Biomedical, Electronic and Telecommunication Engineering, \\ University Federico II of Naples \\ Napoli, Italy \\ E-mail: bracale@unina.it
}

\begin{abstract}
Health Technology Assessment (HTA) is a multidimensional and multidisciplinary methodology, whose aim is to prioritize different concurrent technologies in order to support decision making in healthcare systems. A crucial point in any medical decision is to prioritize clinical, economical and patients' needs on which decision makers base their choices. AHP can improve HTA in this critical task.

In this paper, we applied HTA to assess the effectiveness and the efficacy of Home Monitoring (HM) as a model of continuity of care for patients suffering from congestive heart failure (CHF). We compare HM

\footnotetext{
${ }^{*}$ Corresponding author
}

This study is a part of Ph.D. thesis of the first author, which has been financially supported by an academic award and scholarship for Ph.D., from University Federico II, Napoli. The study has been partially financed also from the Project Remote Health Monitoring (RHM), sponsored by Regione Campania.
\end{abstract}


to other models of care: ambulatory follow-up (benchmark) and Disease Management Programs (DMP). The results lead to conclude that HM is more effective and efficient if compared to the benchmark, but seems to be not cost-effective if compared to DMPs. This conclusion changes when pervasive remote processing is applied to the monitored parameters with the aim to precociously predict critical events. In this case HM seems to be more effective and efficient than the other models of continuity of care. AHP contributed consistently in achieving these conclusions.

Keywords: AHP, HTA, Home Monitoring, healthcare services, Disease Management Programs

\section{Introduction}

Health Technology Assessment (HTA) is a multidisciplinary and multidimensional process useful to evaluate different technologies, alternative and competitive between them. Health technology, according to the definition of WHO, includes also health procedures, and so far services or model of care as HM and DMP. Although there are some works that apply HTA and AHP to support the hospital's purchasing negotiation, there is not such a wide number focusing on the evaluation of new healthcare services. In this paper, we deal with home monitoring of patients suffering from chronic disease, which involves very complex decision tasks and has even macroeconomic consequences.

Congestive Heart Failure (CHF) is a patho-physiological state in which an abnormality of cardiac function is responsible for the failure of the heart to pump blood as really needed by the body. CHF is a strongly degenerative syndrome and age related. Actually CHF prevalence increases rapidly with age, raising from about $3 \%$ in 65 year old patients to more than $11 \%$ in patients over 84 [Mosterd, 1999]. For this reason it is considered as a typical syndrome of the old age, with 74 years [Guidelines, 2003] as mean age of the affected population. The number of patients with CHF is increasing, mainly because of the growing number of elderly people [2000 Heart and Stroke, 1999].

Follow-Up (F-UP) of patients suffering from CHF. F-UP involves long term use of different structures from hospitals to patient's home, and several operators, from specialists to the patient himself and his relatives [Pecchia, 2007]. Disease Management Programs (DMP) [Gonsetha, 2004; Holland 2005] are models of care consisting in coordinated and multidisciplinary healthcare interventions and communications for populations with conditions in which patient self-care efforts are significant [DMAA, 2008]. DMP are mainly oriented to five chronic diseases: ischemic heart disease, diabetes, COPD, asthma and heart failure. DMP generally include telephone calls during which patients interact with trained nursing professionals, and require an extended series of interactions, including a strong educational element. Patients and their relatives are expected to play an active role in managing their own diseases. Home Monitoring (HM) services are models in which the disease management is integrated with remote monitoring of signs, symptoms, physiological parameters and biomedical signals. This implies that HM is, generally, more complex than DMP, requiring more resources, and so far is more expensive. Different articles defined different models of HM, which rise from periodical trans-telephonic transmissions to pervasive systems for continuous monitoring of patients' data. Moreover, there are different strategies for the information's management. The majorities of studies accumulate such information, which is then analyzed by a physician. There are few interesting studies in which remote systems process acquired data in order to individuate precociously destabilizations, which, if not recognized, could bring patient in worse conditions, requiring more complex and expensive cares. Nonetheless, such studies are not yet fully supported by clinical literature or wide RCT.

A wide literature investigates effects of Home Monitoring on outcomes of care of patient suffering from CHF. Also DMP effects have been widely investigated. In almost the totality of study, those models have 
been compared to F-UP, which is considered the benchmark as it is also recommended by international guidelines. Nonetheless, there is not wide literature that compares directly costs and outcomes of HM and DMP.

In this study we investigate the performances of HM versus DMP, in order to asses if it is independently cost-effective when compared with other models of continuity of care and not only with the ambulatory follow-up.

\section{Method}

The whole method followed in this HTA consisted of 4 main steps as described in Figure 1. First, with a field analysis, and throughout expert audit, we individuated the needs (Step A) which have to be satisfied by the models of care. These needs are summarized by clinical outcomes. From literature, we assessed the effectiveness of each method measuring how the models satisfy each need.

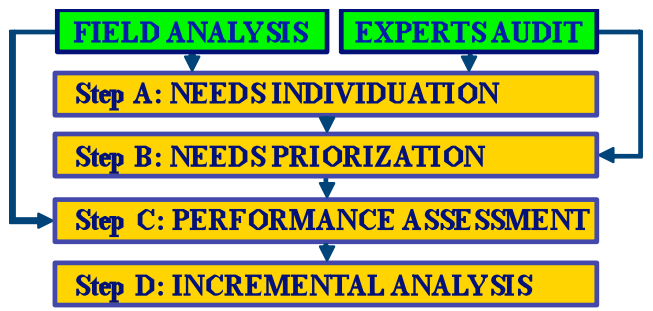

Figure 1. Workflow of the method.

Then all the needs have been prioritized (Step B) by means of AHP [Saaty 1996, Saaty 2005] by submitting scientific questionnaires to experts. As a result of this step we calculated the weights, based on experts' opinion, which expresses relative importance of each need. Finally, we assessed the performance of each model (Step C) by weighting the degree of satisfaction of each need with their relative importance based on experts' opinion.

Since one of the models, compared to the other two, seems to be more effective but also more expensive, we had to perform an incremental analysis (Step D), to evaluate how much it costs to produce "one unit" of outcome with each model of care. Practically we evaluated an incremental Cost-Effectiveness Ratio (iCER) to analytically assess which model is more cost-effective in satisfaction of each need. At last, to assess which model is the most effective and efficient in satisfying all the individuated needs we defined a function of "global utility", using relative importance of each need, and evaluated the incremental CostUtility Ratio (iCUR).

\section{Results and Discussion}

\subsection{Needs individuation and hierarchy definition}

The outcomes more frequently reported in scientific literature have been considered as needs to be satisfied. Current mortality of HF is related to its severity, ranging from 5\% to $10 \%$ in patients with mild symptoms to $30 \%$ to $40 \%$ in severe cases [Massie, 1997]. CHF is a chronic disease; so far a reasonable outcome of the care is to control the stage of pathologies, or at least try to reduce its severity. One of the most diffuse scales to evaluate the severity of pathology is the New York Heart Association (NYHA), which classifies the patients in four classes on the basis of their symptoms. Some authors report that it is possible to regress the patient from a NHYA class to the lower one. For the same reason it is important to improve the quality of life of the patient and his/her relatives.

Furthermore, HF is the leading cause of hospitalisation among the elderly in developed countries [Cowie, 2000; McMurray, 2000]. Moreover, these patients are often re-admitted, up to 50\% within three 
months of initial discharge from hospital [Vinson, 1990, Harjai, 2001]. Luis and al. [Louis, 2003] in their review report that $30 \%$ of patients with a discharge diagnosis of heart failure are readmitted at least once within 90 days and readmission rates range from 25 to 54\% within 3-6 months. Moreover, it may happen that reducing the number of admission and re-admission implies an increasing of annual total hospital staying. One of the reasons is the high number of unattended emergencies, which leads to hospitalize the patient when its condition is worsened to the point to require most long, complex and so far expensive care.

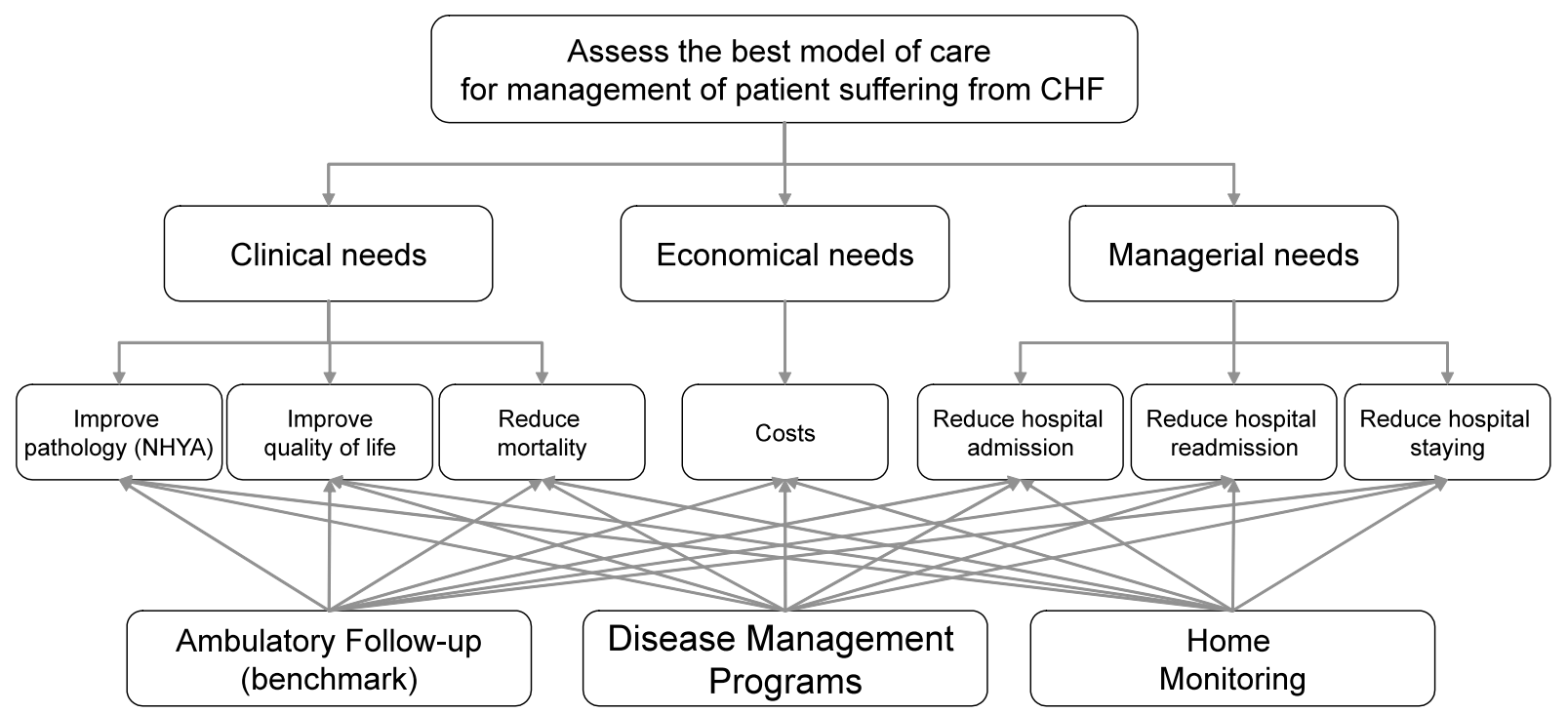

Figure 2. Hierarchy of decision making problem.

All these data implies that in industrialized countries, the direct treatment costs of HF represent $2-3 \%$ of the total health care budget and $10 \%$ of the cardiovascular health care expenditure [Berry, 2001].

\subsection{Needs prioritization}

The questionnaires were submitted to 3 respondents with more then 15 years of experience: 1 professor of health services organization and automation; 1 cardiologist; 1 manager of a health organization. They filled the questionnaires and discuss conclusion achieved reaching consensus. From questionnaires submitted final priority results $(\mathrm{CR}<0.05)$ are reported in the Table 1.

Table 1. Global and relative needs priority.

\begin{tabular}{lccc}
\hline Needs & & \multicolumn{2}{c}{ Priority } \\
& & Global & Relative \\
\hline Improving of pathology (class NHYA) & $\mathrm{w}_{1}$ & 0.80 & $\mathbf{1 0 . 0}$ \\
Reduction of hospital admission & $\mathrm{w}_{2}$ & 0.75 & $\mathbf{9 . 3 8}$ \\
Reduction of hospital readmission & $\mathrm{w}_{3}$ & 0.62 & $\mathbf{7 . 7 5}$ \\
Improving of quality of life & $\mathrm{w}_{4}$ & 0.53 & $\mathbf{6 . 6 3}$ \\
Reduction of mortality & $\mathrm{w}_{5}$ & 0.39 & $\mathbf{4 . 8 8}$ \\
Reduction of hospital staying & $\mathrm{w}_{6}$ & 0.08 & $\mathbf{1 . 0 1}$ \\
Costs & $\mathrm{w}_{7}$ & 0.08 & $\mathbf{1 . 0 0}$ \\
\hline
\end{tabular}


Global Priority, represents the importance of the specific need, compared to the others. Relative Priority is the ratio of "global priority" on the priority of less important need. It says how much each need is important compared to the less important one. The former is used for further analysis, while the latter expresses better the experts' opinion.

\subsection{Performance assessment}

The degree of satisfaction of each need has been assessed by using data coming from meta-analysis [Delli Fraine, 2008; Martınez, 2000; Chaudhry, 2007]. Qualitative results are reported in Table 2.

Table 2: Needs: outcome and output of health care services.

\section{DMP HM [HM]s}

\begin{tabular}{ll:l:l}
\hline Reduction of mortality & + & + & +++ \\
Improving of therapy adherence & + & + & + \\
Improving of phys. parameters adherence & + & ++ & +++ \\
Improving of pathology (class NHYA) & + & + & + \\
Improving of education/lifestyle & + & ++ & ++ \\
Improving of quality of life & + & + & + \\
Reduction of hospital admission & ++ & +++ & +++ \\
Reduction of hospital readmission & ++ & ++ & +++ \\
Reduction of hospital staying & ++ & +++ & ++ \\
\hline +: more than 1.3 times compared to F-up; \\
++: more than 2.5 times compared to F-up; \\
+++: more than 3.0 times compared to F-up; \\
[HM]S: HM improved with remote processing of signals, signs, symptoms
\end{tabular}

We did not find a meta-analysis, which compares HM to DMP, but all compare each to F-UP. Moreover, for some needs we did not find a significant quantification of satisfaction, but just a qualitative description. The data we reviewed lead to conclude that HM and DMP overcome F-UP in all the selected needs. Moreover HM slightly overcomes DMP in: improving patient's "physiological parameters adherence" [Cappuccio, 2005]; improving patient and his relatives "education/lifestyle" [Artinian, 2003]; reducing "hospital staying" mainly because admissions for emergencies are reduced. Instead, HM overcome widely DMP and F-up when signals, signs, symptoms are remotely processed to individuate precociously destabilization of health condition of the patient. Especially Bondmass et al. [Bondmass, 2000] showed that, when data processing is used on transmitted data, there was no unattended emergency. This affects the reduction in number of re-hospitalizations, mortality and also hospital staying because the precocious admission of home visits allows stabilizing the patient. Moreover Hudson et al. [Hudson], enrolling 91 participants, mean age 74, in a Remote Physiological Monitoring (RMP) demonstrate that remote processing of weight and BP has intrinsic value as a tool for early identification of worsening physiological profiles. Basically the conclusion of those studies was that clinicians, alerted by the system, could call the participant and discussing current variations in signs and symptoms: then modulating therapy of diuretics and beta-blockers, they may take corrective measures before patients require emergency care or hospitalization. Nevertheless those interesting results are not yet supported by robust significant data and so far they could not enter this analytic HTA. Finally, we considered the cost of any model of care per patient per year. We selected costs reported by the few articles we found which compare the three models [Jerant, 2001; Whitten, 2002]. In this way we have not data coming from wide 
studies, losing maybe part of generality of the results, but insuring homogeneity of measured costs, which is, in our opinion, a crucial need in a HTA study.

The results we found in literature have been first normalized. Then we calculate the ratio between the data regarding HM and DMP and the data regarding F-UP. All the results are expressed in the Table 3. The first number in each column $\left(\mathrm{E}^{\mathrm{X}}\right)$ indicates the effectiveness of each model normalize per row. The second number ( $\mathrm{R}^{\mathrm{DMP}}$ and $\mathrm{R}^{\text {нм}}$ ) indicate the ratio between each value compared to Follow-up (benchmark). The ratios are much easier to understand and communicate to decision makers. The effectiveness $\left(\mathrm{E}^{\mathrm{x}}\right)$, represent how each model satisfy each need (outcome).

Table 3: costs and effectiveness of the three models normalized per row. For instance the cost of telemedicine is 21.2 times the one of F-UP.

\begin{tabular}{|c|c|c|c|c|}
\hline & & & Effectiven & ness \\
\hline & & F.UP $\mathbf{P}^{*}$ & DMP & HM \\
\hline Outcomes & & $\mathrm{E}^{\mathrm{F} . \mathrm{UP}}$ & $\mathrm{E}^{\mathrm{DMP}} \mathrm{R}^{\mathrm{DMP}}$ & ${ }^{\mathrm{P}} \mathrm{E}^{\mathrm{HM}} \mathrm{R}^{\mathrm{HM}}$ \\
\hline Reduction of hospital admission & $\mathrm{E}_{1}$ & 0.14 & $0.43 \quad 2.8$ & 0.432 .8 \\
\hline Reduction of hospital readmission & $\mathrm{E}_{2}$ & 0.19 & 0.392 .1 & 0.422 .2 \\
\hline Reduction of hospital staying & $\mathrm{E}_{3}$ & 0.15 & $0.39 \quad 2.5$ & 0.463 .0 \\
\hline Reduction of mortality & $\mathrm{E}_{4}$ & 0.28 & $0.36 \quad 1.3$ & $0.37 \quad 1.3$ \\
\hline Improving of pathology (NHYA) & $\mathrm{E}_{5}$ & 0.27 & $0.33 \quad 1.2$ & 0.411 .5 \\
\hline Improving of quality of life & $\mathrm{E}_{6}$ & 0.22 & $0.35 \quad 1.6$ & $0.43 \quad 2.0$ \\
\hline Costs & $\mathrm{C}$ & 0.04 & $0.16 \quad 4.3$ & 0.8021 .2 \\
\hline 1 & & & 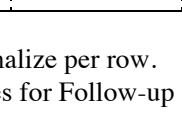 & \\
\hline
\end{tabular}

\subsection{Incremental analysis}

Since Home Monitoring, compared to the other two models, seems to be more effective but also more expensive, it is necessary to recon the incremental Cost-Effectiveness Ratio (iCER).

$$
\text { (i) } \quad i C E R_{\text {Outcome } K K}=\frac{C^{D M P[o r(H M)]}-C^{F . U P}}{E_{\text {Outcome } K}^{D M P(H M)]}-E_{\text {Outcome_K }}^{F . U P}}
$$

The iCER expresses the cost-effectiveness of each model for each outcome because express the cost of each unit of effectiveness added by the model of care, compared with the benchmark. Table 4 shows that DMP is more cost-effective then HM in all the selected outcomes. 
Table 4: incremental Cost-Effectiveness Ratio (iCER) of DMP and HM compared with F-UP. iCER have to be evaluated for each need.

\begin{tabular}{lccc}
\hline iCER & DMP & HM & HM/DMP \\
\hline Reduction of hospital admission & 0.41 & 2.62 & 6.33 \\
Reduction of hospital readmission & 0.60 & 3.30 & 5.51 \\
Reduction of hospital staying & 0.50 & 2.45 & 4.90 \\
Reduction of mortality & 1.50 & 8.44 & 5.63 \\
Improving of pathology (NHYA) & 2.00 & 5.43 & 2.71 \\
Improving of quality of life & 0.92 & 3.62 & 3.92 \\
\hline
\end{tabular}

The third column is the ratio between iCER of DMP and HM in each outcome. This ratio indicates that to improve "one unit" of outcome, HM is from 2.71 to 6.33 times more costly then DMP.

Finally, we defined a function "Utility", weighting each outcome with global priority weigh of each need expressed by experts. Practically we calculate the Utility performing the scalar product of the vector of priorities (Table 1) per the vector of effectiveness (Table 3). Then we estimated the incremental Cost Utility Ratio (iCUR).

$$
\text { (ii) } \quad i C U R=\frac{C^{D M P[\operatorname{or}(H M)]}-C^{F . U P}}{\sum_{i=1}^{6}\left[w_{i} *\left(E_{i}^{D M P[o r(H M)]}-E_{i}^{F-U P}\right)\right]}
$$

The iCUR assesses that one unit of Utility, produced with HM is 5.4 times more costly then if produced with DMP.

Table 1: incremental Cost Utility Ratio (iCUR) of DMP and HM compared with F-UP. iCUR pools together the performance in each need satisfaction.

\begin{tabular}{lccc}
\hline & DMP & HM & HM/DMP \\
\hline iCUR & & & \\
\hline
\end{tabular}

\section{Conclusion}

AHP was extremely useful to quantify decision-makers' opinion. This was necessary in order to define clearly the framework of variables, which influence the HTA of new healthcare services. Moreover, the use of AHP allows defining an objective "Utility Function". This is not an easy task since some times is not clear how each variable contribute to the final aim, if not performing complex sensitivity analysis.

Disease Management Programs and Home Monitoring, benchmarked to ambulatory follow-up, improve all the outcomes and the outputs in the care and follow-up of patients suffering from CHF. HM overcome slightly DMP in satisfaction of some outcomes, but is almost five times more expensive. The iCER, as the iCUR, shows the DMP are more cost-effective than HM. HM may result convenient if benchmarked to ambulatory follow-up, nonetheless it seems to be not absolutely cost-effective when compared to other model of patients' management as DMP. This result could be reversible if remote processing is used to improve HM, to prevent crisis and emergencies. Nonetheless we did not found yet in literature enough data to confirm analytically the advantages of remote processing in preventing destabilizations which, if 
not precociously detected, could bring to hospitalization and to severe conditions. Concluding, from this preliminary results it seems that Telemedicine and Home Monitoring models need to offer new functionalities, such as remote processing finalized to primary prevention, in order to be really competitive.

\section{REFERENCES}

American Heart Association. 2000 Heart and Stroke Statistical Update. Dallas, Tex: American Heart Association; 1999

Artinian NT et al. Pilot study of a Web based compliance monitoring device for patients with congestive heart failure. Heart Lung 2003;32:226-33

Berry C et al. Economics of chronic heart failure. Eur J Heart Fail 2001;3:283-91

Bondmass $\mathrm{M}$ et al. The effect of physiologic home monitoring and telemanagement on chronic heart failure outcomes. Internet J Adv Nurs Pract 2000;3:17

Cappuccio FP et al. Blood pressure control by home monitoring: meta-analysis of randomised trials. BMJ 2004; 329:145.

Cowie MR et al. The epidemiology of heart failure. Eur Heart J 1997; 18:208-25.

Delli Fraine J. L. et al, Home-based telehealth: a review and meta-analysis. Journal of Telemedicine and Telecare, 2008. 14: 62-66

DMAA: The Care Continuum Alliance. DMAA definition of disease management. Retrieved 2008-12-04.

Harjai KJ et al. Simple clinical variables are markers of the propensity for readmission in patients hospitalized with heart failure. Am J Cardiol 2001;87:234-7

Hudson L. R. et al. Remote Physiological Monitoring: Clinical, Financial, and Behavioral Outcomes in a Heart Failure Population. Disease Management. 2005; 8: 372-81

J. Gonsetha et al. The effectiveness of disease management programmes in reducing hospital readmission in older pa-tients with heart failure: a systematic review and meta-analysis of published reports. Eur Heart J 2004;25:1570-1595.

Jerant AF et al. Reducing the cost of frequent hospital admission for congestive heart failure: a randomized trial of a home telecare intervention. Medical Care 2001; 39(11):1234-1245.

L. Pecchia M. Bracale et alt. Technology assessment for evaluating integration of ambulatory follow-up and home monitoring. IFMBE Proceedings, 16(20), 758-761,2007

Louis A. A. et al. A systematicr eview of telemonitoring for the management of heart failure. The European Journal of Heart Failure. 2003. 5:583-590

Martınez Andre's et al, A systematic review of the literature on home monitoring for patients with heart failure Journal of Telemedicine and Telecare 2000. 12:234-241. 
Massie B et al. Evolving trends in the epidemiologic factors of heart failure: rationale for preventive strategies and comprehensive disease management. Am Heart J. 1997;133:703-712

McMurray JJ et al. Heart failure. Epidemiology, aetiology, and prognosis of heart failure. Heart 2000;83:596-602

Mosterd A et al. Prevalence of heart failure and left ventricular dysfunction in the general population. Eur Heart J 1999; 20: 447-55

R Holland et al. Systematic review of multidisciplinary interventions in heart failure. Heart 2005;91:899906.

Saaty T. L., The Fundamentals of Decision Making and Priority Theory with the Analytic Hierarchy Process, Vol. IV of the Analytic Hierarchy Process Series, RWS. 1996.

Saaty T. L., Theory and Applications of the Analytic Network Process: Decision Making with Benefits, Opportunities, Costs and Risks, RWS, 2005

Sarwat I. Chaudhry et al. Telemonitoring for Patients with Chronic Heart Failure: A Systematic Review. J Card Fail. 2007 February ; 13(1): 56-62.

Task Force European Society of Cardiology, Guidelines for the diagnosis and treatment of chronic heart failure Eur Heart 2003, J 22: 1527-1560.

Vinson JM et al. Early readmission of elderly patients with congestive heart failure. J Am Geriatr Soc 1990;38:1290-5

Whitten Pamela $\mathrm{S}$ et al. Systematic review of cost effectiveness studies of telemedicine interventions. BMJ 2002; 324: 1434-37. 